\title{
Epidemiology of healthcare-associated infections in Polish intensive care. A multicenter study based on active surveillance
}

\author{
Michal Walaszeka , Anna Rozanska ${ }^{b}$, Malgorzata Bulanda ${ }^{b}, J^{a d w i g a ~ W o j k o w s k a-M a c h ~}{ }^{b}$, \\ Polish Society of Hospital Infections Teamc
}

\begin{abstract}
Aim. The aim of this study was to evaluate the incidence of health care-associated infections (HAls) in patients treated in Polish intensive care units (ICUs).

Methods. This retrospective analysis was based on the results of active targeted surveillance, according to the recommendation of the ECDC (European Centre of Disease Control and Prevention, HAI-Net light protocol), conducted in 2013-2015 in seven ICUs for adults located in southern Poland (observational study).

Results. The incidence of HAl was $22.6 \%$ and $28.7 / 1000$ person-days (pds). The incidence of pneumonia (PN) was $8.0 \%$, bloodstream infections (BSIs) 7.2\% and urinary tract infections (UTIs) 3.7\%. The incidence per 1000 pds was as follows: PN 10.2, BSIs 9.2 and UTIs 4.7. PN was the most common source of secondary bloodstream infection (45\%); the second was UTIs (22\%). Mortality (directly and indirectly) associated with HAI was $10.8 \%$ and was related to the presence of PN or primary BSIs. HAls were usually (69.2\%) caused by Gram-negative bacteria; Klebsiella spp. and nonfermenting Gram-negative rods demonstrated very high antibiotic resistance.

Conclusion. Despite the lack of widely implemented active targeted surveillance programmes and top-down incentives, it is possible to carry out effective surveillance of HAls in ICUs in Poland. The results of this study are comparable with the ECDC data, but the results are alarmingly high in two fields: epidemiology of PN and BSIs and very high antibiotic resistance in Gram-negative rods, which indicate the need for intense control in this area and for further studies to clarify the source of the observed discrepancy.
\end{abstract}

Key words: infection control, healthcare-associated infections, intensive care units, bloodstream infections, pneumonia, urinary tract infections

Received: October 17, 2017; Accepted with revision: February 22, 2018; Available online: March 1, 2018 https://doi.org/10.5507/bp.2018.006

aPolish Society of Hospital Infections, Krakow, Poland

${ }^{b}$ Department of Microbiology, Faculty of Medicine, Jagiellonian University, Krakow, Poland

'Polish Society of Hospital Infections Team (listed at the end of the article)

Corresponding author: Anna Rozanska, e-mail: a.rozanska@uj.edu.pl

\section{INTRODUCTION}

Among HAIs (healthcare-associated infections) in the ICU (intensive care unit), one can distinguish forms dangerous to patient life, such as PN (pneumonia), BSI (bloodstream infection) and UTI (urinary tract infection). HAI incidence may be associated with many risk factors, such as the use of invasive diagnostic and therapeutic procedures, the environment of treatment (including technological advancement), underlying diseases and comorbidities, which are the reasons for hospital treatment. Traumatic injuries are the current leading cause of hospitalization in intensive care units, they are associated with a high risk of hospital complications (including HAIs) and significantly increased risk of death ${ }^{1,2}$. Even though the constant progress of medicine allows for the treatment of more complex diseases and injuries, it can also generate the risk of HAI (ref. ${ }^{1,3,4}$ ). The development of anaesthetic techniques has increased the age of patients as well as the number of those with serious coexisting diseases qualified for complex surgery and prolonged hospital treatment, which further elevates the risk of hospitalization in the ICU. It is thus particularly important to conduct effective HAI surveillance, with a detailed analysis of HAI incidence in relation to the epidemiological situation of a given department, hospital, region and country.

The differences in rates suggest differences in HAI monitoring complexity and surveillance sensitivity in various countries. In Poland from 1997 to 2001, hospitals could voluntarily participate in the passive surveillance programme conducted by the Polish Society of Hospital Infections (PSHI), which allowed for the ongoing assessment of the epidemiological situation in hospitals and provided an opportunity to work out standards for the effective surveillance and HAI prevention ${ }^{5,6}$. Data from this programme revealed that the HAI incidence in ICUs was $19.72 \%$ (ref. ${ }^{5}$ ). The surveillance currently conducted by the PSHI is based on the assumptions of the HealthcareAssociated Infections Surveillance Network (HAI-Net), which is a European network for the control of HAIs. This network is coordinated by the European Centre for Disease Prevention and Control (ECDC). 
The aim of the study was to analyse the epidemiological situation in Polish intensive care units in terms of healthcare-associated infections based on the example of a multi-centre study conducted using the HAI-Net ICU tools created by the ECDC, with particular emphasis on the following:

- the possibility of conducting continuous surveillance in Polish hospitals and

- comparing the similarities and differences between Polish and European hospitals.

\section{MATERIAL AND METHODS}

This retrospective analysis was carried out based on the results of an observational, prospective active targeted surveillance of hospital infections implemented in 2011 based on the recommendation of the ECDC (HAI-Net light protocol) (ref. ${ }^{7}$ ). This study was conducted from 1 January 2013 to 31 December 2015 in seven ICUs located in southern Poland. All the units participating in the study were a part of non-teaching multi-profile hospitals, with an average number of beds of 414 (range from 224 to 669 ). The average fulltime-equivalent nurse per bed ratio was 2.6. Almost all 6 of the surveyed units were trauma and medical ICUs without cardiac surgery, neurosurgery or oncology (no immunocompromised, haematological and/or transplant patients). Only 1 unit was a surgical ICU without trauma or emergency patients. In terms of ward type and patient characteristics, the study wards were typical Polish ICUs. Care bundles for patients with catheters or ventilated patients and the selective decontamination of the digestive tract were not used.

All study hospitals had infection control teams consisting of epidemiological nurses (no more than one per 200 beds) and a physician as the team leader (their duties related to the study averaged $1 / 5$ th of fulltime-equivalent work); 2 hospitals specialized in medical microbiology.

The diagnosis and classification of infections such as BSI (bloodstream infections), PN (pneumonia), UTI (urinary tract infections) and OTH (other) used definitions developed by the ECDC (ref. ${ }^{7,8}$ ). OTH infections included infection of the bones and joints (BJ), lower respiratory tract infection (LRT), infection of the gastrointestinal tract (GI), genital infection (REPR) and surgical site infection (SSI). Pneumonia was divided into subgroups based on microbiological diagnostics: positive quantitative culture from a minimally contaminated LRT specimen (PN1); positive quantitative culture from a possibly contaminated LRT specimen (PN2); alternative microbiological methods (PN3); other positive culture of sputum or non-quantitative culture of samples from the lower respiratory tract (PN4); and no positive results in microbiological tests (PN5). Bloodstream infections were divided into primary and secondary. Primary infections were distinguished based on the origin of infection: related to central venous catheter (CVC-BSI); associated with peripheral venous catheter (PVC-BSI); arterial catheterrelated (ART-BSI); and unknown source (C-BSI). In addition, primary vascular catheter-related infections were divided into categories based on the microbiological diagnosis: localized infection without positive blood culture (CRI1), generalized infection with positive blood culture (CRI2) and infection with positive blood culture (CRI3). Secondary bloodstream infections were the result of other infectious processes taking place in the body, which occurred when the same microorganism was isolated from blood culture and other focus of infection. Among these conditions, the following types of secondary bloodstream infections were distinguished: secondary to respiratory tract infection (BSI-S-PUL); secondary to urinary tract infection (BSI-S-UTI); secondary to infection of the skin and soft tissues (BSI-S-SST); secondary to the surgical site infection (BSI-S-SSI); secondary to gastrointestinal tract infection (BSI-S-GI); and secondary to other infections (BSI-S-OTH). Based on the microbiological diagnosis, two types of UTI infections were distinguished: microbiologically confirmed symptomatic urinary tract infection (UTI-A) and microbiologically unconfirmed urinary tract infection (UTI-B). Information about patients infected with HAI was collected through active control in accordance with the methodology of surveillance HAI protocol for ICUs, with detailed data collection only for HAI cases $^{7}$. The study excluded patients whose hospital stay in the ICU was shorter than 2 days and those in whom the signs of infection occurred within 2 days from the beginning of hospitalization.

The fatality rate was calculated as the proportion of patients who died from a specified disease among all individuals diagnosed with the disease. The evaluation of this relationship (direct or indirect) was performed by a local infection control team on the basis of the collected medical records.

Based on symptoms and signs, the following clinical materials were collected for microbiological analysis: blood, urine, wound swabs, stool, bronchial aspirate, bronchial alveolar lavage, tips of vascular catheters and others. All supervised hospitals implemented and applied the principles of the European Committee on Antimicrobial Susceptibility Testing (EUCAST).

The de-identified database did not include data on medications (except those used for the treatment of infections) or the details of patients' general status and invasive procedures (such data were not required by the ECDC HAI-Net light protocol used for surveillance) (ref. ${ }^{7}$ ).

Statistical analysis of the material used the statistical program IBM SPSS STATISTICS (Statistical Package for the Social Sciences STATISTICS 24, Armonk, NY, USA and Microsoft Excel Microsoft Office 2016 Redmond, WA, USA) and Microsoft Excel by Microsoft. The level of significance was $P<0.05$. We also calculated odds ratio (OR) and 95\% confidence level.

To compare the incidence of qualitative variants in several populations, the chi-square Pearson test of independence was used. The use of data was approved by the Bioethical Committee of the Jagiellonian University (No. KBET / 122.6120.118.2016 from 25.05.2016). All the data entered into the electronic database and analysed in this study were previously anonymised. 


\section{RESULTS}

Among the 2547 patients hospitalized in study units, HAI were detected in 510 patients ( 189 women and 321 men). The median age of these patients was 63 years, 67 years in women and 60 years in men. The length of stay in the units was 10.3 days on average and 50.9 days in infected patients (median 35.0 days).

The intubation utilization ratio measuring the presence of total patient-days with intubation and calculated by dividing the number of intubation (and ventilator) days by the number of patient days was 0.79 . The centralvenous catheter $(\mathrm{CVC})$ utilization ratio measuring the presence of total patient-days with CVC and calculated by dividing the number of CVC days by the number of patient days was 0.89 The urinary catheter utilization ratio measuring the presence of total patient-days with urinary catheter and calculated by dividing the number of urinary catheter days by the number of patient days was 0.95 .

In the study period, 575 HAI cases were diagnosed, with an incidence of $22.4 \%$. There were $205 \mathrm{PN}$ cases, with an incidence of $8.0 \%$ and 10.2/1000 person-days of hospitalization (VAP incidence: 12.3/1000 ventilatordays); 184 BSIs, with an incidence of $7.2 \%$ and 9.2 persondays of hospitalization (BSI-CVC 3.2/1000 CVC-days); and 95 UTIs, with an incidence of $3.7 \%$ and 4.7 persondays of hospitalization (CA-UTI 4.9/1000 catheter-days). The mortality (directly and indirectly) associated with HAI was $10.8 \%$ ( 62 cases: directly 42 cases, indirectly 20 cases).

In the study population, PN accounted for most of the HAI cases. In connection with the methods of microbiological diagnostics, it was found that in most study ICUs, the material was collected using invasive methods, including in 80 cases of PN1 (39\%). In 87 cases (43\%), no microbiological tests were carried out or materials were used that had scarce diagnostic value (PN4+PN5), despite the fact that the substantial part of cases, including 197 PNs (96\% of the total), were linked to mechanical ventilation. A significant statistical relationship was observed between $\mathrm{PN}$ and mechanical ventilation $(P<0.05)$, the length of stay in the unit $(P<0.001)$ and the age of patients $(P<0.01)$. In the case of pneumonia, the mortality directly and indirectly associated with HAI was $10.7 \%$ (22 deaths, table 1); a significant relationship was found between the presence of PN and death $(P<0.05)$ and between the presence of PN and the coexistence of secondary bloodstream infection $(P<0.001)$-Table 1.

Among all diagnosed HAIs, BSIs were the second most common form of clinical infection and were divided

Table 1. Multivariate analysis of healthcare-associated pneumonia and types of pneumonia.

\begin{tabular}{|c|c|c|c|c|c|c|c|}
\hline \multirow{3}{*}{$\begin{array}{l}\text { Factor } \\
\text { category }\end{array}$} & \multirow{2}{*}{$\begin{array}{l}\text { Total } \\
\mathrm{n}(\%)\end{array}$} & \multicolumn{5}{|c|}{ Pneumonia by microbiological diagnosis } & \multirow{3}{*}{$P^{*}$} \\
\hline & & $\begin{array}{l}\text { PN } 1 \\
\mathrm{n}(\%)\end{array}$ & $\begin{array}{l}\text { PN } 2 \\
\mathrm{n}(\%)\end{array}$ & $\begin{array}{l}\text { PN } 3 \\
\mathrm{n}(\%)\end{array}$ & $\begin{array}{l}\mathrm{PN} 4 \\
\mathrm{n}(\%)\end{array}$ & $\begin{array}{l}\text { PN } 5 \\
\mathrm{n}(\%)\end{array}$ & \\
\hline & $205(100)$ & $80(39)$ & $26(12.7)$ & $12(5.9)$ & $63(30.7 \%)$ & $24(11.7)$ & \\
\hline \multicolumn{7}{|c|}{ Mechanical ventilation } & \multirow{3}{*}{$<0.05$} \\
\hline Yes & $197(96.1)$ & $76(37.1)$ & $25(12.2)$ & $11(5.4)$ & $63(30.7)$ & $22(10.7)$ & \\
\hline No & $8(3.9)$ & $4(2.0)$ & $1(0.5)$ & $1(0.5)$ & $0(0.0)$ & $2(1.0)$ & \\
\hline \multicolumn{7}{|c|}{ Days of hospitalization } & \\
\hline $2-7$ & $11(5.4)$ & $1(0.5)$ & $0(0.0)$ & $1(0.5)$ & $2(1.0)$ & $7(3.4)$ & \multirow{4}{*}{$<0.001$} \\
\hline $8-14$ & $23(11.2)$ & $9(4.4)$ & $3(1.5)$ & $4(2.0)$ & $4(2.0)$ & $3(1.5)$ & \\
\hline $15-21$ & $32(15.6)$ & $20(9.8)$ & $4(2.0)$ & $0(0.0)$ & $6(2.9)$ & $2(1.0)$ & \\
\hline 22 & $139(67.8)$ & $50(24.4)$ & $19(9.3)$ & $7(3.4)$ & $51(24.9)$ & $12(5.9)$ & \\
\hline \multicolumn{7}{|c|}{ Coexistence of secondary bloodstream infections } & \multirow{3}{*}{$<0.001$} \\
\hline PUL - yes & $15(7.3)$ & $8(3.9)$ & $0(0.0)$ & $0(0.0)$ & $7(3.4)$ & $0(0.0)$ & \\
\hline UTI - yes & $30(14.6)$ & $6(2.9)$ & $13(6.30$ & $1(0.5)$ & $5(2.4)$ & $5(2.4)$ & \\
\hline \multicolumn{7}{|c|}{ Death, relationship } & \\
\hline Direct & $16(7.8)$ & $5(2.4)$ & $1(0.5)$ & $0(0.0)$ & $6(2.9)$ & $4(2.0)$ & \multirow{4}{*}{$<0.05$} \\
\hline Indirect & $6(2.9)$ & $2(1.0)$ & $2(1.05)$ & $0(0.0)$ & $2(1.0)$ & $0(0.0)$ & \\
\hline Without & $6(2.9)$ & $5(2.4)$ & $0(0.0)$ & $1(0.5)$ & $0(0.0)$ & $0(0.0)$ & \\
\hline Total & $28(13.7)$ & $12(5.95)$ & $3(1.5)$ & $1(0.5)$ & $8(3.9)$ & $4(2.0)$ & \\
\hline
\end{tabular}

n, number of patients; PN - pneumonia; LRT - lower respiratory tract; PN1 - positive quantitative culture from minimally contaminated LRT specimen; PN2 - positive quantitative culture from possibly contaminated LRT specimens; PN3 - alternative microbiology methods. PN4 - other positive sputum culture or non-quantitative LRT specimen culture; PN5 - no positive microbiology; $P^{*}$ level of significance of independence chisquare Pearson test; PUL - bloodstream infections secondary to respiratory tract infection; UTI - bloodstream infections secondary to urinary tract infection 
into 97 (53\%) primary and 87 (47\%) secondary cases. Among primary BSI, patients with central vascular catheter dominated, with 57 cases (59\%), as seen in Table 2.

In the majority of these cases, i.e., $46(70 \%)$, blood cultures were positive (CRI-3); in 7 cases (11\%), changes were topical without positive blood culture (CRI-1); and in 13 cases $(20 \%)$, the infection was generalized without positive blood culture (CRI-2). The relationship was observed between the presence of primary bloodstream infection and the length of stay in the unit $(P<0.01)$. In case of primary bloodstream infections, mortality directly or indirectly related to BSI was $16.5 \%$ (16 deaths, Table 1), and a significant relationship was demonstrated between the presence of primary BSI and death $(P<0.05)$, while the CRI mortality directly or indirectly related to HAI was slightly higher, at $19.7 \%$ (13 deaths). PN was the most common source of secondary bloodstream infection (BSI-S-PUL; 39 cases, 45\%). The other frequent sources of secondary bloodstream infection included UTI ( $19 ; 22 \%)$, SST (10; 12\%), OTH (7; 8\%), GI (6; 7\%) and SSI $(6 ; 7 \%)$. A significant correlation was observed between the presence of secondary BSI, the length of stay in the unit $(P<0.001)$ and the age of patients $(P<0.01)$. Urinary tract infections (UTIs) mainly occurred in patients with bladder catheters: 94 cases $(99 \%)$. These infections in large part (91 cases $(97 \%)$ ) were diagnosed using microbiological methods. Statistical significance was demonstrated between the presence of UTI, bladder
Table 2. Multivariate analysis of the incidence of primary bloodstream infections (BSI) and types of primary bloodstream infections.

\begin{tabular}{|c|c|c|c|c|}
\hline \multirow{3}{*}{$\begin{array}{l}\text { Factor } \\
\text { category }\end{array}$} & \multirow{2}{*}{$\begin{array}{c}\text { Primary } \\
\text { BSI } \\
\text { n (\%) }\end{array}$} & \multicolumn{2}{|c|}{ Type of vascular catheter } & \multirow{3}{*}{$P^{*}$} \\
\hline & & $\begin{array}{c}\text { CVC-BSI } \\
\mathrm{n}(\%)\end{array}$ & $\begin{array}{c}\text { Other catheters } \\
n(\%)\end{array}$ & \\
\hline & $97(100)$ & $57(59.0)$ & $40(41.2)$ & \\
\hline \multicolumn{4}{|c|}{ Length of stay } & \multirow{5}{*}{$<0.01$} \\
\hline 2-7 days & $4(4.1)$ & $2(2.1)$ & $2(2.1)$ & \\
\hline 8-14 days & $14(14.4)$ & $6(6.2)$ & $8(8.2)$ & \\
\hline 15-21 days & $10(10.3)$ & $8(8.2)$ & $2(2.1)$ & \\
\hline 22 days & $69(71.1)$ & $41(42.3)$ & $28(28.8)$ & \\
\hline \multicolumn{4}{|c|}{ Death, relationship } & \multirow{5}{*}{$<0.05$} \\
\hline Direct & $8(8.2)$ & $7(7.2)$ & $1(1.1)$ & \\
\hline Indirect & $8(8.2)$ & $5(5.2)$ & $3(3.1)$ & \\
\hline without & $7(7.2)$ & $3(3.1)$ & $4(4.1)$ & \\
\hline Total & $23(23.7)$ & $15(15.5)$ & $8(8.2)$ & \\
\hline
\end{tabular}

$\mathrm{n}$ - number of patients; CVC-BSI - central vascular catheter; $\mathrm{P} * 1$ level of significance of independence chi-square Pearson test.

Table 3. Bacterial aetiology of Healthcare-Associated Infections in study intensive care units.

\begin{tabular}{|c|c|c|c|c|}
\hline Laboratory confirmed HAI & $\begin{array}{c}\mathrm{PN} \\
\mathrm{n}(\%)\end{array}$ & $\begin{array}{c}\text { BSI Primary } \\
\mathrm{n}(\%)\end{array}$ & $\begin{array}{c}\text { UTI } \\
\text { n }(\%)\end{array}$ & $\begin{array}{l}\text { Total } \\
\mathrm{n}(\%)\end{array}$ \\
\hline Gram-positive cocci & $32(19.0)$ & $36(46.2)$ & $22(24.2)$ & $90(27.6)$ \\
\hline Staphylococcus aureus & $24(14.3)$ & $10(12.8)$ & $6(6.6)$ & $40(44.4)$ \\
\hline CNS & $1(0.6)$ & $12(15.4)$ & $0(0.0)$ & $13(14.4)$ \\
\hline Enterococcus spp. & $2(1.2)$ & $7(9.0)$ & $6(6.6)$ & $15(16.7)$ \\
\hline Streptococcus pneumoniae & $5(3.0)$ & $7(9.0)$ & $10(11.0)$ & $22(24.4)$ \\
\hline Enterobacteriaceae & $83(49.4)$ & $21(26.9)$ & $39(42.9)$ & $143(43.9)$ \\
\hline Citrobacter spp. & $1(0.6)$ & $0(0.0)$ & $0(0.0)$ & $1(0.7)$ \\
\hline Enterobacter spp. & $6(3.6)$ & $2(2.6)$ & $0(0.0)$ & $8(5.6)$ \\
\hline Escherichia coli & $33(19.6)$ & $5(6.4)$ & $16(17.6)$ & $54(37.7)$ \\
\hline Klebsiella spp. & $6(3.6)$ & $9(11.5)$ & $18(19.8)$ & $33(23.1)$ \\
\hline Proteus spp. & $27(16.1)$ & $1(1.3)$ & $5(5.5)$ & $33(23.1)$ \\
\hline Serratia spp. & $10(6.0)$ & $4(5.1)$ & $0(0.0)$ & $14(9.8)$ \\
\hline Nonfermenting Gram-negative bacteria & $37(22.0)$ & $13(16.7)$ & $21(23.1)$ & $71(21.8)$ \\
\hline Acinetobacter spp. & $19(11.3)$ & $7(9.0)$ & $0(0.0)$ & $26(36.6)$ \\
\hline Pseudomonas aeruginosa & $12(7.1)$ & $5(6.4)$ & $17(18.7)$ & $37(52.1)$ \\
\hline Stenotrophomonas maltophilia & $2(1.2)$ & $0(0.0)$ & $4(4.4)$ & $6(5.4)$ \\
\hline Haemophilus spp. & $4(2.4)$ & $1(1.3)$ & $0(0.0)$ & $5(7.0)$ \\
\hline Other & $11(6.5)$ & $8(10.3)$ & $3(3.3)$ & $22(6.7)$ \\
\hline Total & $163(100.0)$ & $78(100.0)$ & $85(100.0)$ & $326(100.0)$ \\
\hline
\end{tabular}


catheterization $(P<0.001)$ and the length of stay in the unit $(P<0.001)$. In patients treated in ICUs, there were 91 cases of other healthcare-associated infections: 45 (49\%) GI; 20 (22\%) SYS; 15 (16\%) REPR; 3 (3\%) LRI; 3 (3\%) SST; 3 (3\%) SSI; and 2 (2\%) BJ. The clinically relevant material collected from the patients treated in the ICU and diagnosed with healthcare-associated infection contained 493 microorganisms (85.7\%), and no aetiological agent of infection was detected in 82 cases (14.3\%). HAIs were usually (341 (69.2\%)) caused by Gram-negative bacteria. This group included Enterobacteriaceae (179 $(36.3 \%)$ ) and Gram-negative non-fermenting rods (162 $(32.9 \%))$. Gram-positive bacteria caused 132 healthcareassociated infections (26.8\%). Escherichia coli was the most commonly grown species among Gram-negative bacteria (67 (13.6\%)), while Staphylococcus aureus was the most common among Gram-positive bacteria (61 $(12.4 \%))$, as seen in Table 3. Significant drug resistance was found with respect to Klebsiella spp. and nonfermenting Gram-negative bacilli (Table 4). The ESKAPE pathogens (Enterococcus faecium, Staphylococcus aureus, Klebsiella spp., Acinetobacter baumannii, Pseudomonas aeruginosa, and Enterobacter species) represented $48.8 \%$ of all microorganisms.

\section{DISCUSSION}

This is the first report of active targeted surveillance conducted in a population of patients treated in ICUs in Poland and in Central Europe. Based on the metaanalysis carried out by Allegranzi et al. ${ }^{9}$ the risk of HAIs among patients hospitalized in the ICU is $30 \%$. Simonetti et al. $^{3}$ reported an HAI incidence in the ICU of $16 \%$. The one-centre study conducted in Poland by Wieder-Huszla ${ }^{10}$ found the incidence in the ICU to be $24 \%$. Another study carried out in Poland by Kübler et al. ${ }^{11}$, who reported that the incidence in the ICU was $24 \%$, with an incidence density of $22 \%$.

In our study, the median age of hospitalized patients with HAI was 63 years. The results of this study are similar to those conducted in other European countries ${ }^{12}$. HAI-related mortality in Polish ICUs in 1998 accounted for $26 \%$ (ref. $^{6}$ ). We find the fact that the current HAIrelated mortality in the ICUs was lower, at $11 \%$, to be very positive. This value is smaller than the mortality noted in the ECDC report of 2012, where the mean mortal-

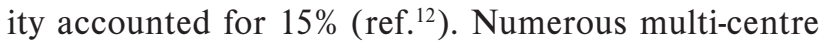
studies confirm the consistency of these results with the outcomes presented in this study ${ }^{11-14}$. However, a detailed data review indicates differences in the HAI epidemiology between Polish ICUs and the HAI-Net data, which may be related to the organization of work and the system of

Table 4. Antimicrobial resistance in microorganisms isolated from HAI (data presented only for species with number of isolated $>25$ ).

\begin{tabular}{|c|c|c|c|}
\hline Bacterial aetiology of HAI & & Antibiotic or resistant codes & NS n $(\%)$ \\
\hline \multicolumn{4}{|l|}{ Gram-positive cocci } \\
\hline \multirow[t]{2}{*}{ Staphylococcus aureus } & 46 & MRSA & $4(8.7)$ \\
\hline & & ampicylin & $4(8.7)$ \\
\hline \multirow[t]{3}{*}{ Enterococcus spp. } & 27 & amoxicilin/ clavulanic acid & $2(7.4)$ \\
\hline & & vancomycin & $2(7.4)$ \\
\hline & & ceftazidim & $2(7.4)$ \\
\hline \multicolumn{4}{|l|}{ Enterobacteriaceae } \\
\hline \multirow[t]{4}{*}{ Escherichia coli } & 38 & ESBL & $3(7.9)$ \\
\hline & & amoxicilin/ clavulanic acid & $14(36.8)$ \\
\hline & & ceftazidim & $4(10.5)$ \\
\hline & & cefotaxim / ceftriaxone & $3(7.9)$ \\
\hline \multirow[t]{4}{*}{ Klebsiella spp. } & 80 & ESBL & $44(55.0)$ \\
\hline & & amoxicilin/ clavulanic acid & $61(76.3)$ \\
\hline & & ceftazidim & $54(67.5)$ \\
\hline & & cefotaxim / ceftriaxone & $56(70.0)$ \\
\hline \multicolumn{4}{|c|}{ Nonfermenting Gram-negative Bacilli } \\
\hline \multirow[t]{3}{*}{ Acinetobacter spp. } & 92 & imipenem & $37(40.2)$ \\
\hline & & meropenem / doripenem & $28(30.4)$ \\
\hline & & sulbactam & $27(29.3)$ \\
\hline \multirow[t]{5}{*}{ Pseudomonas aeruginosa } & 34 & amoxicilin/ clavulanic acid & $1(2.9)$ \\
\hline & & cefotaxim / ceftriaxone & $9(26.5)$ \\
\hline & & imipenem & $10(29.4)$ \\
\hline & & meropenem / doripenem & $10(29.4)$ \\
\hline & & piperacilin & $13(38.2)$ \\
\hline
\end{tabular}

HAI - Healthcare-Associated Infections; NS - non susceptible; n NS - number of NS isolates; MRSA - methicilinresistant Staphylococcus aureus; ESBL - extended spectrum beta-lactamases 
HAI surveillance. In our study, PN was the most common form of HAI, with an incidence of $8 \%$. In the first ECDC study (2007) ( ref. $^{12}$ ) conducted in 55988 patients treated in the ICU in different European countries, the incidence was $7 \%$. Therefore, it was comparable to that presented in this study. However, in the later ECDC report of 2013, the PN incidence was lower and amounted to $5 \%$ (ref. ${ }^{15}$ ). Additionally, reports from Germany on the implementation of the programme KISS indicate the possibility of reducing the PN risk in the units actively involved in continuous infection surveillance ${ }^{16}$. A review of the literature found that in other Polish multi-centre studies, the PN incidence in the ICU varied considerably, i.e., from $2 \%$ to $21 \%$, and the incidence density ranged from 10 to 53 per 1000 patient-days ${ }^{17}$. In the single-centre study conducted in 2010-2014 in ICUs, Wałaszek et al. ${ }^{2}$ reported a PN incidence of $5 \%$, with an incidence density of 7/1000 hospitalizations. In the single-centre studies conducted in the ICU in Wrocław, the PN incidence was 15\% (2007-2010) and $8 \%$ (2014) (ref. ${ }^{11,18}$ ).

Unfortunately, a high incidence of PN was observed in Poland not only in adults ICUs but also in neonatal $\mathrm{ICUs}^{19}$.

In our present study, the PN mortality was $11 \%$. European studies demonstrated that the PN-related mortality was high and amounted to $31 \%$ (ref. $^{12}$ ). In addition, in a single-centre study carried out in Poland, PN mortality in the ICU was $33 \%$ (ref. $^{2}$ ). A much lower PN mortality of $13 \%$ was reported by Duszyńska et al. ${ }^{18}$ in a single-centre study conducted in Poland, and in the study of Wójkowska-Mach et el., the VAP mortality rate was $18 \%\left(\right.$ ref. ${ }^{20}$ ). In a study of 6284 patients, Melsen et al. (ref. ${ }^{21}$ ) demonstrated a PN mortality of $13 \%$. It is difficult to compare the results of our studies, other Polish studies and those conducted elsewhere in the world because a vast discrepancy in the percentage of mortality can result from the use of different criteria for qualification of patient death as associated or not associated with PN. Thus, these results should be considered reliable, with small differences in individual trials. In the later part of our research, in connection with diagnostic methods, the most commonly identified types of pneumonia were PN1 and PN4, while PN3 was the least frequent. Similarly, in the ECDC report ${ }^{12}$, PN1 and PN4 were the most frequent, while PN3 was the least frequent.

In our studies, BSI was the second most common form of HAI, with an incidence of $7 \%$. Unfortunately, the Polish results are twice as high as those obtained in the European programme of infection surveillance, where these rates were as follows: an incidence of $4 \%$ in 2007 (ref. ${ }^{12}$ ) and 3\% in 2014 (ref. ${ }^{2}$ ). However, it should be noted that there are significant differences in the incidence between individual European countries. The sensitivity of the system used to detect infections with particular emphasis on microbiological tests is probably an important factor differentiating individual countries in terms of BSI detection. Importantly, in the currently studied ICUs, the incidence of primary BSI was $4 \%$, while it was $2 \%$ in the study conducted in Poland in 1998 (ref. ${ }^{6}$ ). According to the ECDC data ${ }^{12}$, the percentages of BSI in relation to the source of infection were more varied than in our study and were as follows: $77 \%$ related to the central catheter (vs. 59\% in Poland), 14\% associated with arterial catheter and $9 \%$ associated with peripheral catheter. In this study, the incidence of central catheter-related BSI was $4 \%$ and was higher than in the ECDC study - 3\% (ref. $^{22}$ ). On the other hand, the proportion of ICU beds in Polish hospitals is lower than in most European countries, accounting for 6.9 beds per 100,000 capita of population compared with European averages of 11.5 beds $^{23}$. As a result, Polish ICUs treat only the most seriously ill patients, who have significant risk of infections.

As for another division of vascular catheter-related infections (CRIs), which is based on microbiological diagnostics, the literature review found that the results of our analysis probably indicated the insignificant recognition of vascular catheter-related local infections compared to other European reports in which the proportion of CRI varied between countries and was as follows (on average): CRI 1 (38\% vs. $11 \%$ in Poland), CRI2 (20\% vs. $20 \%$ in Poland) and CRI3 (41\% vs. $70 \%$ in Poland) (ref. ${ }^{9}$ ). In the study group, the mortality of patients with primary BSI was $24 \%$.

In the study conducted by Wójkowska-Mach et al. ${ }^{6}$ in 1998 in Poland, the mortality associated with primary BSI in the ICU was slightly lower and amounted to $19 \%$. The analysis of the secondary BSI incidence found differences in the incidence of infections of different sources, particularly for UTI (ECDC $13 \%$ vs. $22 \%$ in Poland), GI (25\% vs. $7 \%$ in Poland) and PUL (40\% vs. $45 \%$ in Poland) (ref. ${ }^{12}$ ). The third most common form of healthcare-associated infection was urinary tract infection (UTI); according to Duszyńska et al. ${ }^{24}$, the incidence of UTI in single-centre studies was $7 \%$ and was $92 \%$ in patients with urinary catheters. Our study obtained slightly lower values, with an incidence of UTI of 4\% (incidence density 5) and with association with the urinary catheter in $99 \%$ of these infections.

The observed mortality in patients with UTIs was $18 \%$. In another study conducted in Poland in 1998, the UTI mortality in the ICU was similar and amounted to $19 \%$ (ref. ${ }^{6}$ ). These results corresponded to the mortality achieved in the European study, where this rate was $22.8 \%$ (ref. $^{12}$ ). The presented research results come from the first multi-centre study conducted through the active continuous infection surveillance in Polish ICUs. They confirm the possibility of implementing this type of surveillance in Poland. The outcomes can be the basis for an in-depth analysis of the epidemiological situation in intensive care units and the exploration of factors influencing HAI incidence in those departments and thus contribute to the development of more effective preventive actions. This should be the most important element of HAIs surveillance because the epidemiological situation in Polish hospitals differs from that described in HAI-Net ICU ECDC reports. Analysing the data from the pilot study of HAI-Net "standard version" in Polish ICUs allows us to draw the conclusion that the sensitivity of surveillance 
does not depend on the type of protocol chosen and that "the light" version is easier and less time absorbing for HAI surveillance.

The presented study has some limitations. The first is that it was conducted in a relatively small group of seven ICUs. Most of these ICUs hospitalized patients in similar conditions, but no detailed information about comorbidities and no APACHE score or any other scale were assigned to patients. Another limitation is that, although all units used the same protocol, there was no external validation of infection detection. Additionally, the protocol did not require gathering detailed data on invasive procedures (catheters and mechanical ventilation), there were no unique standards of cooperation with microbiology labs, and isolated strains were not banked (hence no detailed characteristics of aetiological factors were available).

\section{CONCLUSIONS}

1. Comparison of healthcare-associated infections incidence in the study ICUs with the literature allows us to conclude that the epidemiological situation in the study units did not significantly vary from situations described in the literature.

2. The incidence of primary and secondary bloodstream infections, which is higher than in many European countries, indicates the need for intensification of control in this area and undertaking further studies to clarify the source of this discrepancy. The main problems were still infections in patients with a central vascular catheter.

3. Differences in the incidence of vascular catheterrelated bloodstream infections may be associated with various microbiological diagnostics in patients with vascular catheters, more careful diagnosis and classification of CRI1 infections, with a tendency to diagnose BSI based on general symptoms or positive blood culture.

4. Comparison of secondary sources of bloodstream infections revealed a greater percentage of infections originating from the urinary tract and pneumonia and a smaller percentage of infections with the source in the gastrointestinal tract.

5. The results of the surveillance carried out under the programme of the PSHI are comparable with the outcomes of the control conducted by the ECDC. This suggests that despite the lack of reports on the implementation of active targeted surveillance programmes and top-down incentives, it is possible to carry out effective surveillance of HAIs in ICUs, even in Poland.

6. Polish ICUs have very high antibiotic resistance in Gram negative rods, especially Klebsiella spp. and nonfermenting Gram-negative bacilli.

\section{ABBREVIATIONS}

ART-BSI, Arterial catheter-related bloodstream infections; ESBL, Extended spectrum beta-lactamases; BJ,
Bones and joints infections; BSI, Bloodstream; C-BSI, Bloodstream infections of unknown source; BSI-S, Bloodstream infections secondary to; CI, Confidence Interval; CNS, Coagulase negative staphylococci; CRI1, Localized infection without positive blood culture; CRI2, Generalized infection with positive blood culture; CRI3, Infection with positive blood culture ; CVC, Central venous-catheter; ECDC, European Centre for Disease Control and Prevention; EUCAST, the European Committee on Antimicrobial Susceptibility Testing; GI, Gastrointestinal infections; HAI , Hospital acquired infections; Infection; ICU, Intensive Care Unit; LRT, Lower respiratory tract infections; MRSA, Methicillin resistant Staphylococcus aureus; N, Number; NS, Non susceptible; OR, Odds Ratio; OTH, Other infection types; pds, Person-days of hospitalization; $P$-value, Probability value; PN, Pneumonia; PN1, Positive quantitative culture from a minimally contaminated LRT specimen; PN2, Positive quantitative culture from a possibly contaminated LRT specimen; PN3, Alternative microbiological methods; PN4, Other positive culture of sputum or non-quantitative culture of samples from the lower respiratory tract; PN5, No positive results in microbiological tests; PSHI, Polish Society of Hospital Infections; PUL, Bloodstream infections secondary to respiratory tract infection; PVCBSI, Bloodstream infections associated with peripheral venous catheter; REPR, Genital infections; SST, Infection of the skin and soft tissues; UTI, Uurinary tract infections; UTI-A, Microbiologically confirmed symptomatic urinary tract infection; UTI-B, Microbiologically unconfirmed urinary tract infection.

\section{Polish Society of Hospital Infections team}

Joanna Domańska, Institute of Theoretical and Applied Informatics, Polish Academy of Sciences in Gliwice; Grzegorz Dubiel Center of Pulmonology and Thoracic Surgery in Bystra, Bystra, Poland Joanna Liberda, Henryk Klimontowicz Specialistic Hospital in Gorlice; Agnieszka Misiewska-Kaczur, Voivodeship Hospital in Bielsko-Biała; Marzena Lech, Hospital in Staszów; Marta Wałaszek, Voivodeship Hospital im. St. Łukasz in Tarnów.

\section{REFERENCES}

1. Prin M, Li G. Complications and in-hospital mortality in trauma patients treated in intensive care units in the United States, 2013. Inj Epidemiol 2016;4:3-18.

2. Wałaszek M, Kosiarska A, Gniadek A, Kołpa M, Wolak Z, Dobroś W, Siadek J. The risk factor for hospital-acquired pneumonia in the intensive care unit. Przegl Epidemiol 2016;70:15-20.

3. Simonetti A, Ottaiano E, Diana MV, Onza C, Triassi M. Epidemiology of hospital-acquired infections in an adult intensive care unit: results of a prospective cohort study. Ann Ig 2013; 25(4):281-9.

4. Ling ML, Apisarnthanarak A, Madriaga G. The Burden of HealthcareAssociated Infections in Southeast Asia: A Systematic Literature Review and Meta-analysis. Clin Infect Dis 2015;60(11):1690-9.

5. Wójkowska-Mach J, Jeljaszewicz J, Bulanda M, Adamski P, Heczko PB. Ocena częstości występowania zakażeń szpitalnych w polskich szpitalach w 1998 roku na podstawie wyników programu Polskiego Towarzystwa Zakażeń Szpitalnych. Przegl Epidemiol 2000;54(1):3744. 
6. Wójkowska-Mach J, Różańska A, Kuthan R, Bulanda M, Heczko P. Mortality associated with hospital acquired infections. Analysis based upon data from the Polish National Program for Control of Infection in 1998. Przegl Epidemiol 2000;54:281-90.

7. ECDC. European surveillance of health care associated infections in intensive care units - HAI-Net ICU protocol, version 1.02. Stockholm: ECDC; 2015. http://ecdc.europa.eu/en/publications/publications/ healthcare-associated-infections-hai-icu-protocol.pdf (accessed 4.10.2016).

8. ECDC. Point prevalence survey of healthcare-associated infections and antimicrobial use in European acute care hospitals - protocol version 4.3. Stockholm: ECDC; 2012. http://ecdc.europa. eu/en/publications/publications/0512-ted-pps-hai-antimicrobial-use-protocol. pdf. (accessed 4.10.2016).

9. WHO: Report on the burden of endemic health care- associated infection worldwide. A systematic review of the literature. http:// apps.who.int/iris/bitstream/10665/80135/1/9789241501507_eng. pdf (accessed 4.10.2016).

10. Wieder-Huszla S. Monitorowanie zakażeń szpitalnych w oddziale intensywnej terapii medycznej; Pomeranian J Life Sci 2010;56(3):209.

11. Kübler A, Duszyńska D, Rosenthal VD, Fleischer M, KaiserT, Szewczyk T, Barteczko-grajek B. Device-associated infection rates and extra length of stay in an intensive care unit of a university hospital in Wroclaw, Poland: International Nosocomial Infection Control Consortium's (INICC)findings. J Crit Care 2012;27:105-10.

12. ECDC. Surveillance of healthcare-associated infections in Europe, 2007. Stockholm: ECDC;2012:43-71. http://ecdc.europa.eu/en/ publications/Publications/120215_SUR_HAI_2007.pdf (accessed 4.10.2016)

13. Agodi A, Auxilia F, Barchita M, Brusaferro S, D'Alessandro D, Grillo OC, Montagna MT, Pasquarella C, Righi E, Tardivo S, Torregrossa V, Mura I; GISIO-SITI. Trends, risk factor and outcomes of healthcare- associated infections within the Italian network SPIN-UTI. J Hospit Infect 2013;84:52-8.

14. Gords B, Vrijens F, Hulstaest F, Devriese S, Van de Sande S. The 2007 Belgian national prevelence survey for hospital-acquired infections. J Hospit Infect 2010;75:163-7.

15. ECDC. Point prevalence survey of health care associated infections and antimicrobial use in European acute care hospitals. Stockholm:
ECDC; 2013:186-7 http://ecdc.europa.eu/en/publications/publications/healthcare-associated-infections-antimicrobial-use-pps.pdf (accessed 4.10.2016).

16. Gastmeier P, Schwab F, Sohr D, Behnke M, Geffers C: Reproducibility of the surveillance effect to decrease nosocomial infection rates. Infect Control Hosp Epidemiol 2009;30(10):993-9.

17. Rosenthal VD, Maki DG, Salomato R, Moreno CA, Mehta Y, Higuera F, Cuellar LE, Arikan OA, Abouqal R, Leblebicioglu H. DeviceAssociated Nosocomial Infections in 55 Intensive Care Units of 8 developing Countries. Ann Intern Med 2006;145:582-91.

18. Duszyńska W, Rosental VD, Dragan B, Węgrzyn P, Mazur A, Wojtyra P, Tomala A, Kübler A. Ventilator-associated pneumonia monitoring according to the INICC project at one center. Anesthesiology Intensive Therapy 2015;47(1):34-9.

19. Wójkowska-Mach J, Merritt TA, Borszewska-Kornacka M, Domańska J, Gulczyńska E, Nowiczewski M, Helwich E, Kordek A, Pawlik D, Adamski P. Device-associated Pneumonia of Very Low Birth Weight Infants in Polish Neonatal Intensive Care Units. Adv Med Sci 2016;61:90-5.

20. Wójkowska-Mach J, Bulanda M, Różańska A, Kochan P, Heczko PB. Hospital acquired pneumoniain the intensive care units in Polish hospitals. Infect Control Hosp Epidemiol 2006; 27(7):784-6.

21. Melsen WG, Rovers MM, Groenwold RH, Bergmans DC, Camus C, Bauer TT, Hanisch EW, Klarin B, Koeman M, Krueger WA, Lacherade JC, Lorente L, Memish ZA, Morrow LE, Nardi G, van Nieuwenhoven CA, O'Keefe GE, Nakos G, Scannapieco FA, Seguin P, Staudinger T, Topeli A, Ferrer M, Bonten MJ. Attributable mortality of ventilator-associated pneumonia: a meta-analysis of individual patient data from randomised prevention studies. Lancet Infect Dis 2013;13(8):665-71.

22. ECDC. Annual epidemiological report 2014. Antimicrobial resistance and healthcare-associated infection, Stockholm ECDC; 2015. http://ecdc.europa.eu/en/publications/publications/antimicrobialresistance-annual-epidemiological-report.pdf (accessed 4.10.2016).

23. Rhodes A, Ferdinande P, Flaatten H, Guidet B, Metnitz PG, Moreno RP. The variability of critical care bed numbers in Europe. Intensive Care Med 2012;38:1647-53.

24. Duszyńska W, Rosenttal VD, Szczęsny A, Woźnica E, Ulfik K, Ostrowska E, Litwin A, Kübler A. Urinary tract infections in intensive care unit patients - a single center, 3-year observational study according to the INICC project. Anesthesiology Intensive Therapy 2016;48(1):1-6. 